\title{
THE AETIOLOGY AND DIAGNOSIS OF HAEMORRHAGIC DISEASES IN CHILDREN
}

\author{
By A. D. M. JACKson, M.D., M.R.C.P., D.C.H. \\ Assistant Medical Registrar, The Hospital for Sick Children, Great Ormond Street, London
}

The subjects of haemostasis and of the haemorrhagic diseases are the cause of much speculation and controversy. Many theories have been put forward to explain both the physiological and the pathological processes concerned. It is proposed in this review to include an account of those theories which are most widely accepted at the present time.

\section{The Physiology of Haemostasis}

Haemostasis may be defined as the physicochemical process by which the flow of blood from injured vessels is arrested. For the sake of simplicity it may be described in a series of stages:

I. The walls of the capillaries in the injured area contract and considerably diminish the flow of blood. Small amounts of thromboplastin (thrombokinase) are liberated by the injured tissues, but not in sufficient quantity to affect coagulation significantly.

2. Platelets collect in and around the breach in the vessel wall, partly as a mechanical stop-gap, but mainly so that they may, by clumping and disintegrating in this abnormal environment, release a substance known as the platelet factor, which, because of its theoretical role in the clotting mechanism, has also been called thromboplastinogenase (Quick, 1949).

3. The platelet factor activates a plasma factor, to which Quick (1947) has given the name thromboplastinogen and thromboplastin is formed. Thromboplastin from this source effects the formation of thrombin from the plasma prothrombin complex, which includes prothrombin, calcium, and at least one other substance variously termed the "labile factor,' 'factor $V$ ' and 'accelerator factor' (Stefanini, 195I). A chain reaction is now set up by thrombin, which is thought to facilitate further disintegration of platelets: more thromboplastin is therefore formed, more prothrombin is converted into thrombin, and so on. The whole purpose of this chain reaction is to produce maximal amounts of thrombin as rapidly as possible.

4. In the presence of thrombin, circulating fibrinogen is converted into fibrin. With the appearance of the fibrin clot in the wound and in the damaged vessels the chain reaction is halted owing to the fact that fibrin inhibits further formation of thrombin.

5. The capillary walls are now beginning too dilate after their initial constriction (Macfarlane, I94I), but the presence of the fibrin clot and itso attachment to the vessel wall, brought about by the adhesive properties of the platelets, prevent any further flow of blood through the gap. The subsequent retraction of the clot-again the result of some physical property of the platelets-serves to complete the process by bringing the capillary walls together and holding them there while the gap is repaired and the debris of coagulation is removed (Quick, 1942).

It is difficult to understand why intravascular coagulation does not normally take place in intact vessels. The fundamental reason for this is probably that, where there is no break or other abnormality of the capillary wall, the platelets will not disintegrate and no platelet factor will be released. Other factors, such as heparin, which acts as an anti-thrombin, may also play some part in the prevention of thrombosis.

It appears, then, that two main mechanisms are involved in haemostasis: the vascular mechanism and the coagulation mechanism. Normally these two systems are complementary and efficient haemostasis cannot be achieved if either breaks down. In certain circumstances, however, the coagulation mechanism is redundant. This is the case with fine puncture wounds where there is little trauma and the break in the capillary wall is so fine that vascular contraction, aided only by the physical action of the platelets, is sufficient to close it. On the other hand, efficient coagulation 
is by itself incapable of arresting haemorrhage if the vascular mechanism is defective.

\section{Classification of the Haemorrhagic Diseases}

From the preceding account it will be observed that several individual factors are intimately concerned with haemostasis. They may be enumerated thus:

I. The vascular mechanism:

Capillaries.

2. The coagulation mechanism:

Platelets and platelet factor.

Plasma thromboplastinogen.

Plasma prothrombin.

Plasma fibrinogen.

The haemorrhagic diseases occurring in childhood can be classified according to which of these factors is considered to be at fault.

Disorders of the Vascular Mechanism

(a) Congenital defect of the capillaries:

Hereditary haemorrhagic telangiectasia.

(b) Mechanical rupture of the capillaries, e.g. pertussis.

(c) Avitaminosis:

Scurvy.

(d) Non-thrombocytopenic purpura:

I. Anaphylactoid.

2. Symptomatic, e.g. infections, acute nephritis.

Disorders of the Coagulation Mechanism

(a) Platelet or platelet factor deficiency:

I. Thrombocytopenic purpura:

(i) Symptomatic, e.g. bone marrow disease, infections.

(ii) Idiopathic.

(In idiopathic purpura there is also a capillary defect.)

2. Hereditary haemorrhagic thrombasthenia.

(b) Thromboplastinogen deficiency:

Haemophilia.

(c) Prothrombin deficiency:

I. Haemorrhagic disease of the newborn.

2. Idiopathic.

(d) Fibrinogen deficiency:

Congenital afibrinogenaemia.

The term purpura simplex has been omitted from this classification deliberately, because it is vague and is often loosely used to describe mild types of purpura whatever their aetiology.

\section{Diagnostic Tests}

Diagnosis in the haemorrhagic diseases depends to a great extent on the results of certain investigations, the principles and application of which will now be discussed.

Tourniquet test (Hess's capillary resistance test).
This clinical test is designed to demonstrate $a \varrho$ lowered resistance of the capillary wall to internal pressure. It is performed by applying a blood $\stackrel{\varrho}{C}$ pressure cuff to the upper arm and maintaining $a \stackrel{.}{\Rightarrow}$ pressure midway between the systolic and diastolic $\stackrel{0}{9}$ blood pressures for at least five minutes. The $\overline{0}$ appearance, under these conditions, of more than ten petechial haemorrhages in a circle of skin $\frac{\bar{c}}{\frac{F}{a}}$ $5 \mathrm{~cm}$. in diameter, with its centre $4 \mathrm{~cm}$. below the $\stackrel{\mathbb{Q}}{\mathscr{Q}}$ bend of the elbow, implies a weakness of the capillary wall. Various modifications of the test ${ }^{\infty}$ have been suggested with the idea of improving its. accuracy and obtaining a quantitative measure of $\vec{\omega}$ the capillary defect, but these methods are not $\stackrel{\omega}{\sigma}$ reliable and in practice the test remains crude and inaccurate. It is often, but not invariably, positive 3 . in the diseases due to a vascular defect, but in the coagulation defects it is always negative. In $\omega$ idiopathic thrombocytopenic purpura it is almost $\vec{A}$ always positive.

Platelet count. Accuracy in platelet counts is difficult to achieve, whether the direct or indirect 음 method is used, but fortunately thrombocytopenia, $\vec{C}$ when it is present, is usually considerable. The $D$ normal platelet count is from 250,000 to $500,000 \frac{\pi}{\square}$ per $\mathrm{cmm}$. with a wide daily variation. In the new $=\frac{1}{3}$ born the count is normally much lower $(150,000$ to 250,000 per $\mathrm{cmm}$.) and rises to the higher figures during the first three months of life (Tocanting o 1938). A repeatedly normal count excludes the diagnosis of thrombocytopenia, which is said to exist when the platelets fall below the arbitrary figure of 100,000 per $\mathrm{cmm}$., although most cases of idiopathic thrombocytopenic purpura have counts below 50,000 per $\mathrm{cmm}$. In children spon- $\stackrel{\mathbb{Q}}{\varrho}$ taneous haemorrhage usually arises when the plate- $\overrightarrow{\vec{A}}$ let count falls to 70,000 per $\mathrm{cmm}$., but haemorrhage $\frac{0}{3}$ may also occur with higher counts and, conversely, there may be no bleeding even though the platelet? count is below 70,000 per cmm. Therefore in haemorrhagic conditions associated with thrombo- 3 . cytopenia the bleeding is not necessarily related to the level of the platelet count.

Clot retraction. It was pointed out earlier that 8 clot retraction depends on the physical activity of $₹$ the platelets. A quantitative deficiency (below about 70,000 per $\mathrm{cmm}$.), and possibly also a quali- $\rightarrow$ tative defect of the platelets, will, therefore, lead to a failure of clot retraction or at least to a prolonged clot retraction time. Normal blood, when allowed $\%$ to clot in a test tube, shows retraction of the clot $N$ with the expression of a small quantity of clear $N$ serum within one hour, and if then the volumes of the clot and the serum are determined a quantita- 0 tive estimate of clot retraction may be obtained $\underset{\Phi}{\widetilde{T}}$ (Macfarlane, 1939). The error in estimating $\operatorname{clot} \stackrel{\oplus}{+}$ retraction in vitro is considerable, but in idiopathic 0 thrombocytopenic purpura the defect is usually $\frac{\vec{D}}{\mathbb{D}}$ 
severe and there is a complete failure of the clot to retract. In haemophilia, when the clot eventually. forms, it retracts normally.

Bleeding time. This simple procedure measures the time taken for the flow of blood from a small puncture wound to cease. Using the single puncture technique in the lobe of the ear, the normal bleeding time is between two and five minutes and appears to depend both on the ability of the capillary wall to constrict after injury and on the presence of a normal number of platelets. Since both capillaries and platelets are unaffected in haemophilia the bleeding time is essentially normal in this condition, despite a gross defect of the coagulation mechanism. Even in diseases due to a vascular defect, if the platelet count is normal, the bleeding time will also be normal, and it is only in idiopathic thrombocytopenic purpura that it is invariably prolonged.

Coagulation time. This test determines the time taken for blood to clot after removal from the body and is an assessment of the efficiency of the coagulation mechanism. In children the capillary tube method of Dale and Laidlaw is convenient, since only a drop or two of blood from a finger prick is needed. The normal coagulation time by this method is not greater than three minutes (five minutes in the newborn) and abnormally long times are found in haemophilia and the prothrombin deficiency diseases. In afibrinogenaemia the blood does not coagulate. The coagulation defect in thrombocytopenic purpura (i.e. platelet factor deficiency) is not demonstrable by this test, a fact which will be explained in the next paragraph.

Prothrombin consumption test. It has been thought for some time that a coagulation defect must exist in thrombocytopenic purpura, despite the fact that the coagulation time is normal, and the prothrombin consumption test was designed by Quick (1947) to demonstrate this defect. The principle of the test is simple. In the presence of an optimum amount of thromboplastin large quantities of prothrombin are converted into thrombin during coagulation. There is, therefore, very much less prothrombin in serum than in plasma. Since only small amounts of thrombin are necessary to produce enough fibrin for the coagulation time to be normal, a relative deficiency of thromboplastin may exist without prolonging the coagulation time. The amount of prothrombin consumed in this case will be less than normal and the amount left in the serum will be considerable. The prothrombin consumption test measures the amount of prothrombin consumed during the coagulation of a sample of blood and an estimate of the available thromboplastin in that sample is thus obtained. It has already been stated that plasma thromboplastin is derived from the interaction of platelet factor with thromboplastinogen, so that in thrombocytopenic purpura, where there is a deficiency of the former, and in haemophilia, where there is a deficiency of the latter, the consumption of prothrombin during clotting should be low. In practice this is found to be the case and the prothrombin consumption test provides some confirmation of the theories of aetiology in these two diseases. From a practical point of view, the test is not suitable for routine use and, in any case, does not provide sufficient information to make it useful in diagnosis.

Prothrombin estimation. By ensuring the presence of an excess of thromboplastin, calcium and fibrinogen the coagulation time of a sample of blood becomes a direct measure of the quantity of prothrombin it contains and the various methods of estimating prothrombin depend on this principle. In practice, however, there are many technical complications which may influence the result, so that the test gives only a rough indication of prothrombin concentration. The normal prothrombin time is between $I_{5}$ and 25 seconds, but the result is usually compared with that of a sample of normal blood (tested under identical conditions) and expressed as a percentage of the normal (prothrombin index). The value of the prothrombin estimation lies in detecting diseases of the pro- 8 thrombin deficiency group. In all the othe haemorrhagic diseases the prothrombin concentrate tion is normal.

Fibrinogen estimation. The fibrinogen content of normal blood is from 0.2 to $0.4 \mathrm{gm}$. per cent., but it is rarely necessary to carry out this estimation, as all the cases of afibrinogenaemia so far recorded have shown a complete absence of fibrinogen, resulting in a failure of the blood to clot. It is possible, however, that unusual forms of the disease may occur and the fibrinogen should be estimated where the cause of haemorrhagic symptoms is obscure.

\section{Aetiology and Diagnosis}

Hereditary haemorrhagic telangiectasia. This condition is inherited as a Mendelian dominant and affects both sexes. It is due to a developmental defect in some of the small vessels of the skin and mucous membranes, the affected vessels being thin walled and dilated. The onset of symptoms is usually delayed until late childhood or early adult life, but may occur as early as two years. Bleeding from the mucous membranes arises spontaneously and epistaxis is the commonest symptom. The skin vessels do not bleed spontaneously and petechial haemorrhages are not seen. The visible telangiectases on the face, in the mouth and nose, and under the nails appear only in adult life, but isolated 'spider naevi' uncon- 


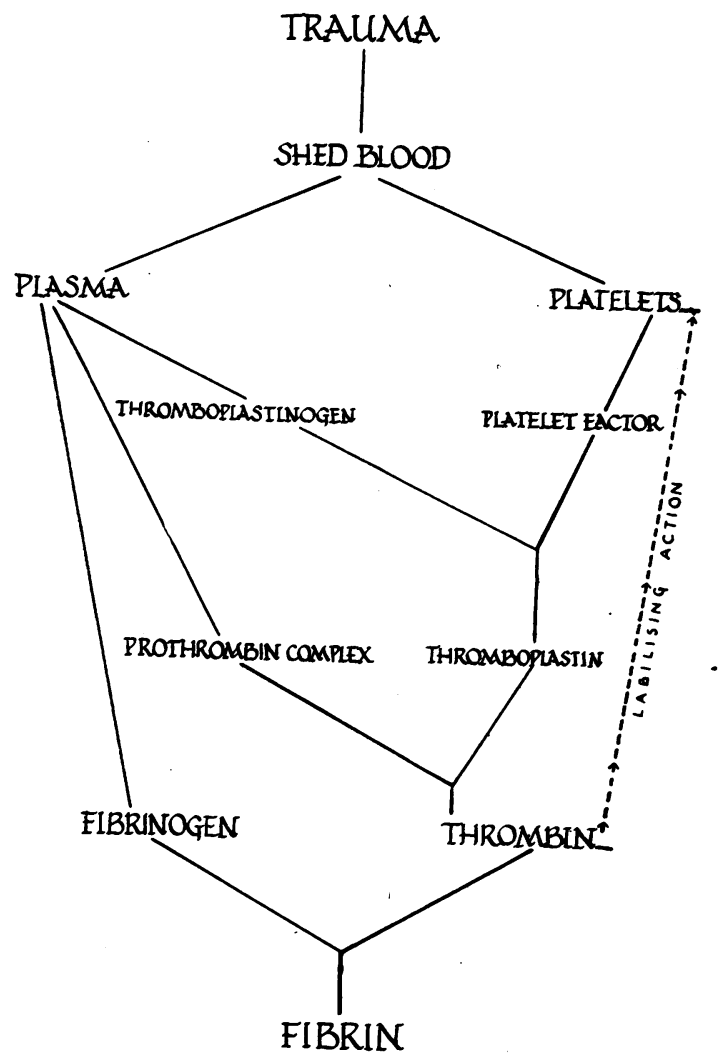

FIG. I.-The coagulation mechanism.

nected with this disease are often seen in children. Since all the diagnostic tests are normal and there is usually a family history, diagnosis from other haemorrhagic diseases is not difficult, but when the bleeding is confined to haemoptysis, haematemesis or haematuria detailed investigation may be necessary to exclude other local causes of these symptoms.

Scurvy. A deficiency of vitamin C results in a grave disturbance of ossification and an increase in the permeability of the capillaries which leads to spontaneous haemorrhages. The age incidence of the disease is between six months and two years, the majority of cases occurring at the end of the first year. It is rarely seen in breast-fed babies, and in artificially-fed babies it occurs only if there have not been adequate supplements of vitamin $C$.

After a period of vague symptoms the main clinical features appear. There are haemorrhages in the skin in the form of petechiae or ecchymoses and there is usually some haematuria and subperiosteal haemorrhage. The gums, where teeth have erupted, become red, swollen and spongy, and the changes in the bones produce beading of the ribs and extreme tenderness of the limbs with pseudo-paralysis. In addition, there are pathognomonic radiological changes, most marked in the long bones, including generalized rarefaction; the dense metaphyseal ' white line' of calcified cartilage with a localized zone of rarefaction proximal to it; a similar dense line ringing the epiphyses and, in the later stages, calcification in the subperiosteal haemorrhages (Fig. 2). The diagnostic tests are all normal except for the tourniquet test, which is often positive. The diagnosis of scurvy is thus easily established by a combination of the typical clinical features, the radiological appearances and the normal blood findings. When there is still any doubt, the amount of ascorbic acid excreted in the urine after a test dose may be estimated and this will be low in a case of scurvy.

There is no convincing evidence that either vitamin $P$ or the similar compound, rutin, play any part in the causation of scurvy or of any other condition associated with increased capillary fragility (Lancet, 1950).

Non-thrombocytopenic purpura. A number of diverse clinical conditions are grouped together under this heading, all of them showing purpuric eruptions and often other forms of haemorrhage, without platelet deficiency. The cause of bleeding in these cases is an increased fragility of the capillary wall. Two main groups are recognized the anaphylactoid group, which has a distinctive clinical picture, and a group in which the purpure is only a symptomatic manifestation of some primary disease.

The term anaphylactoid purpura is used to cover the various disorders in which the capillary permeability is considered to be allergic in nature, resulting from sensitivity, mainly to bacterial infection, but possibly also to other exciting factors. The separation of these disorders into different syndromes, such as allergic purpura, Henoch's purpura and Schönlein's purpura, is unnecessary, since they are all fundamentally due to the same cause and differ only in the severity or the site of the symptoms. In anaphylactoid purpura, then, there is often a history of a preceding bacterial infection, such as a streptococcal sore throat, and the presenting symptoms are a rash, acute abdominal symptoms with melaena, and painful nonhaemorrhagic swellings in and around the joints of the limbs. These main symptoms are usually all present together, but the severity of one or all of them may be extremely variable. Acute nephritis is a common complication (Gairdner, 1948). The rash has a characteristic appearance and distribution. It is primarily maculo-papular with a haemorrhagic component superadded, but true petechiae and ecchymoses are not common. The typical distribution is on the buttocks, the extensor surfaces of the limbs and around the ankles (Fig. 3). 


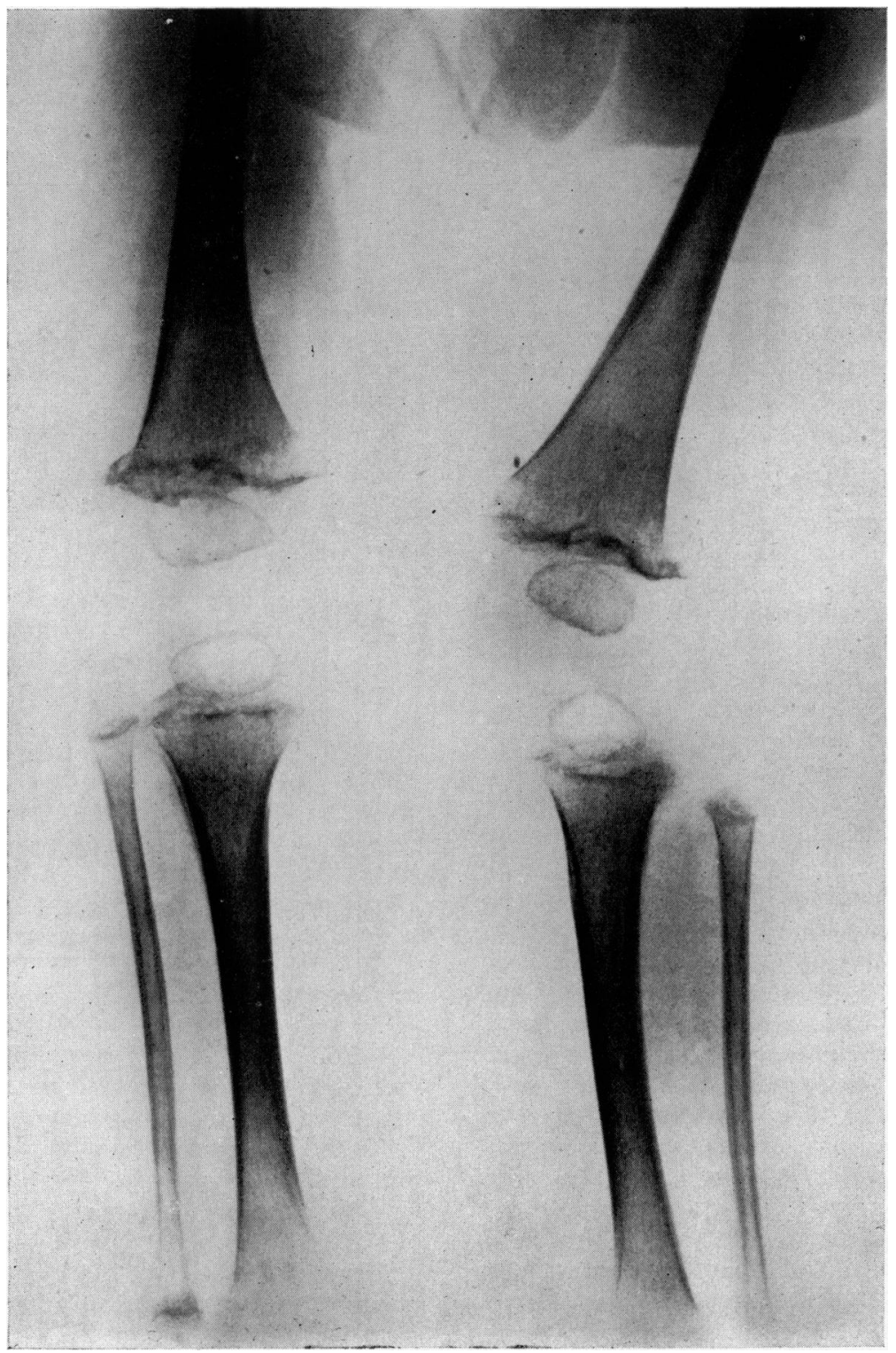

Fig. 2.-Radiological appearances in scurvy. There are dense lines of calcification in the metaphyses and surrounding the rarefied epiphyses. The localized zones of rarefaction adjacent to the metaphyseal lines are well shown in the femora. Calcification of a sub-periosteal haematoma is commencing at the upper ends of the tibiae. 
There are no abnormal findings in the blood in this group, although the tourniquet test is occasionally positive, and the appearance of the capillaries in the nail bed as seen under a microscope is essentially normal. The similarity between the abdominal symptoms of anaphylactoid purpura and those of intussusception is well known, but the presence of a rash or joint swellings, or both, will usually help to differentiate the two conditions. Other causes of painful, swollen joints must also be excluded on clinical grounds, but the skin lesions are usually unmistakable. When there is a true purpuric eruption the normal platelet count will exclude the thrombocytopenic purpuras, but not the non-thrombocytopenic group, which is secondary to other diseases. Thercfore history taking and examination must be aimed at eliminating such diseases which include the later stages of the acute specific fevers, subacute bacterial endocarditis, meningococcal infections and acute nephritis. In these diseases, which produce a toxic effect on the capillary wall, bleeding is merely a minor incident and is usually confined to the petechial haemorrhages in the skin. Where purpuric haemorrhages occur as a result of mechanical pressure, as in the paroxysms of whooping cough, the cause will be obvious.

Non-thrombocytopenic purpura in the newborn is a symptom of septicaemia or asphyxia (where the capillary weakness is due to anoxia), and may also be the result of mechanical compression during labour. The differential diagnosis of this special group will be discussed in the section on haemorrhagic disease of the newborn.

Thrombocytopenic purpura. The haemorrhagic condition associated with thrombocytopenia is most commonly secondary to some other disease. The thrombocytopenia in this case is due to a depression of the megakaryocytes in the bone marrow and the bleeding is probably due to a combination of the platelet deficiency and a capillary weakness also brought about by the underlying disease. This situation occurs, for example, in leukaemia (Fig. 4), aplastic anaemia, the reticuloses, and drug intoxication with such drugs as arsenic, gold and sulphonamides. In addition, most of the acute infections, which have already been described as causing a vascular defect alone, may also cause thrombocytopenia by their toxic effect on the bone marrow and, in children particularly, simple upper respiratory infections may produce a similar effect. In most of these conditions the haemorrhagic manifestations are relatively unimportant compared with the main features of the primary disease, but it is obvious that in any case of thrombocytopenic purpura full clinical and haematological investigations must be carried out in order to establish, if possible, a

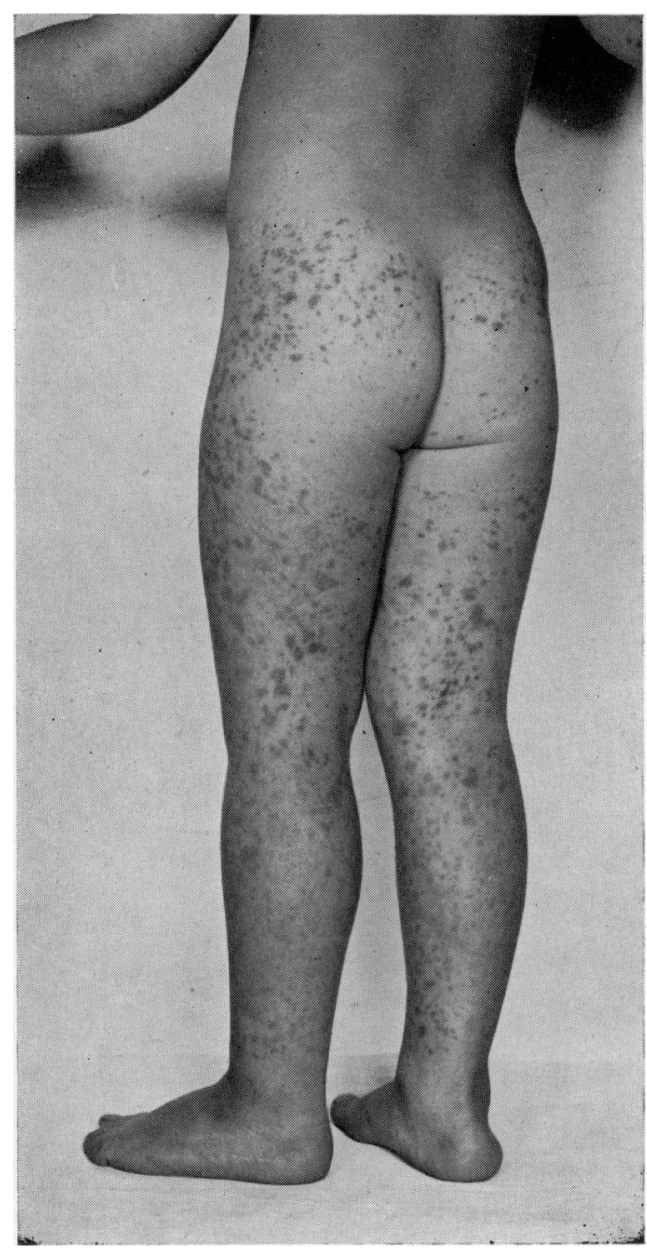

FIG. 3.-The rash in anaphylactoid purpura.

cause for the low platelet count. When no cause whatever can be demonstrated, the condition may be termed idiopathic thrombocytopenic purpura or purpura haemorrhagica.

This disease is commoner in females than in males and only about 30 per cent. of cases occur under the age of ten, the highest incidence being in young adults. Occasionally babies born of mothers who are suffering from the disease may develop temporary thrombocytopenic purpura during the first few days of life, suggesting the passage of some causative factor across the placenta. The aetiology of the condition remains obscure. All that can be said at present is that there is a platelet (and therefore platelet factor) deficiency, possibly due to an inhibition of megakaryocyte maturation, combined with a capillary defect. Exactly how these factors are related to each other and to the cause of the disease is unknown, but, since 


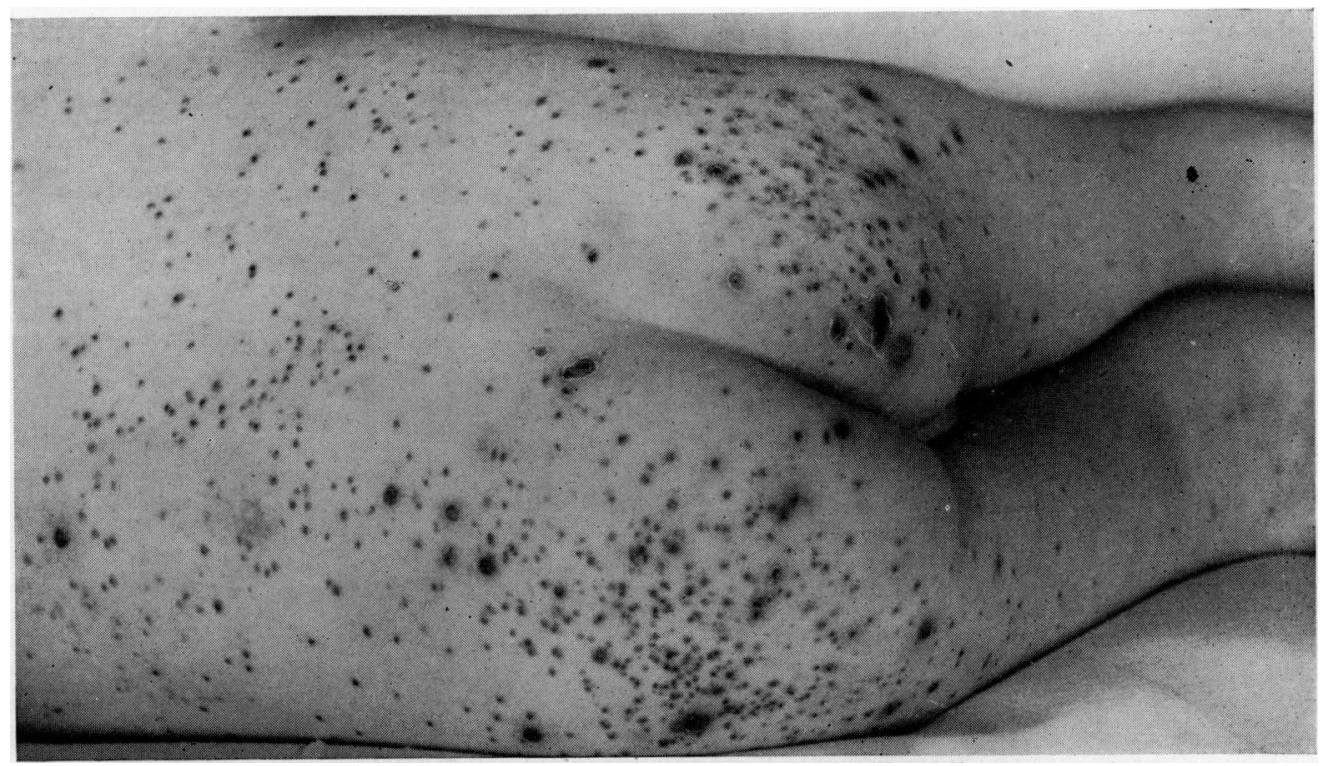

FIG. 4.-Symptomatic purpura. Skin haemorrhages from a rase of chronic myeloid leukaemia with a platelet count of 65,000 per c.mm.

removal of the spleen often cures the symptoms, it has been suggested that some substance is produced by the splcen which acts on both the marrow and the capilla: ies to produce the observed defects.

Idiopathic thrombocytopenic purpura is characterized by haemorrhages, either spontaneous or induced by mild trauma, into the skin and from the mucous membranes. Skin petechiae and bruises are particularly common and the bleeding from any part of the body may be slight or dangerously severe. The spleen is occasionally palpable and capillary microscopy shows abnormal branching and distortion of the capillaries in the nail bed (Macfarlane, I94I). The platelet count is always low, usually below 50,000 per $\mathrm{cmm}$., the bleeding time is prolonged, clot retraction is defective and the tourniquet test is usually positive, but many of the cases are recurrent with variable periods of remission, during which these abnormal findings may revert to normal. The coagulation time is normal and megakaryocytes are present in the bone marrow in normal quantities. By means of these tests it is usually a simple matter to exclude other haemorrhagic diseases, but difficulty may be encountered in deciding whether the thrombocytopenia is secondary or not when the suspected primary cause is of a mild or trivial nature. However, although investigations of the blood may give identical results in both the idiopathic and the symptomatic groups, the finding of a decreased number of megakaryocytes in the marrow will exclude the formcr. Any case of thrombocytopenic purpura with an anaemia more severe than could be accountcd for by blood loss must be considered as leukaemia until this diagnosis is disproved by examination of the bone marrow.

Hereditary haemorrhagic thrombasthenia (Glanzmann's disease). This title is one of many (e.g. pseudo-haemophilia, von Willebrand's disease) given to a group of rare haemorrhagic disorders of which the aetiology is quite unknown. In some of the cases it is thought that there is an excessive stability of the platclets which leads to a deficiency of platelet factor, and the findings of Macfarlane (I94I) on capillary microscopy in this disease suggest that there is a primary capillary defect. As far as recorded cases are concerned, the condition appears to be hereditary and affects both sexes. Haemorrhage may occur, usually as a result of some slight injury, at any age and may be of varying severity. Glanzmann (1949) himself states that ecchymoses and bleeding from the mucous membranes are common, but that he has not known haemarthrosis to occur, and he maintains that the constant finding in the blood is a defective clot retraction. There is no thrombocytopenia; in 
fact the platelet count is often higher than normal, and the bleeding and coagulation times are not prolonged. Very rarely cases have been described in which the clot retraction is normal but the bleeding time is prolonged, and to this group the name of von Willebrand has been attached. Diagnosis is really a matter of exclusion of the well-recognized diseases and, for the present at least, the miscellaneous remainder must be placed in this group. However unsatisfactory this may seem, it is probably less confusing than attempting to separate each unusual case or group of cases into svndromes with cponymous titles.

Therc are some individuals, often with similarly affected relatives, who appear to bleed more than normal, although not severely, after dental extractions or tonsillectomy. There is, however, never any demonstrable defect in the vessels or in the coagulation mcchanism in these cases and there seems no reason to connect them with thrombasthenia. Whether this sort of bleeding, the existence of which is based solely on clinical impressions, is within the range of normal or is due to some defect which present-day in vitro tests are too crude to detect will no doubt be decided in the future.

Haemophilia. This disease is inherited by means of a sex-linked recessive gene and affects males whose mothers are heterozygous carriers of the defect. Sporadic cases may appear occasionally, presumably as a result of gene mutation, but no proven casc has been recorded in a female. The nature of the coagulation defect in haemophilia is still uncertain, but the modern theories which attempt to explain it must come very near to the truth. lt is reasoned that, since fibrin is not formed adequately and the fibrinogen is normal, a thrombin deficiency exists. The prothrombin complex is also normal, so that the fault lies with thromboplastin, a fact which is confirmed by the lowered consumption of prothrombin during the coagulation of haemophilic blood. As thromboplastin formation depends on the platelet factor and the plasma factor, thromboplastinogen, one or both of these factors must be ultimately responsible for the abnormality of coagulation. With regard to the platelets, it has been demonstrated by Merskey (1950) that the low prothrombin consumption in thrombocytopenia can be remedied by the addition of platelets from haemophilic blood, and he also showed that the similar defect in haemophilia can be corrected by normal plateletfree plasma. In other words, in haemophilia the platelets are normal and the missing factor is contained in normal plasma. By a process of elimination, therefore, a deficiency of thromboplastinogen appears to be the cause of the disease. Thromboplastinogen is probably identical with anti-haemophilic globulin, the plasma fraction which has been described by Lewis et al. (1946) and which they stated would correct the prolonged $\frac{\varrho}{c}$ coagulation time in haemophilia. A circulating anti-coagulant has also been described in some $\stackrel{\rho}{\rightarrow}$ cases of haemophilia, but this is a rare occurrence and often only appears after repeated transfusion.

The bleeding in haemophilia is initiated by $\stackrel{\mathbb{Q}}{\varrho}$ trauma, which may only be trivial, and is not in common under the age of about six months. Its onset is delayed a little after the injury, but its duration is considerably prolonged. Any part of $\vec{\omega}$ the body may be affected but the subcutaneous tissues and muscles, the tongue and tooth sockets are the common sites. Petechial haemorrhages do not occur, but haemarthrosis, which is rare in $N$ other haemorrhagic disorders, is often seen and leads eventually to ankylosis of the affected joints. Episodes of bleeding often recur at regular intervals and the coagulation time, although nearly always prolonged, may vary with a similar periodicity. Apart from the low prothrombin consumption all the other diagnostic tests are normal. The diagnosis is made on the family history, the clinical features and the laboratory findings. In the rare cases in which the coagula tion time is normal, confirmation of the diagnosis may be obtained from the prothrombin con sumption test (Merskey, 195I).

Haemorrhagic disease of the newborn. Haemorrhage is not uncommon in the newborn period and occurs in some 5 per cent. of babies. Such easily recognized causes as trauma or infection account for the majority of cases and only a few (about one in 500 live births) are associated with an abnormally low level of prothrombin in the blood and appear to have no other obvious cause. To the latter group the term haemorrhagic disease of the newborn is applied. The prothrombin level in all newborn babies falls to about 30 per cent. of normal by the second day of life; the formation of prothrombin in the liver depends on the presence of vitamin $K$, and during the first few days of life there is no intake of this vitamin and there are no intestinal organisms to synthesize it; therefore the prothrombin lost into the placenta immediately after birth is not replaced. By the fourth or fifth day these factors are no longer operating, and by the seventh day the prothrombin has returned to normal. This physiological hypoprothrombinaemia is not usually associated with bleeding, but it may be; and while the majority of infants with haemorrhagic disease have an unusually low prothrombin level (below 20 per cent.), these levels may occasionally exist without symptoms. The concentration of prothrombin is not therefore entirely responsible for the bleeding in this 
disease and the existence of some additional factor, as yet undetermined, must be postulated.

The bleeding in haemorrhagic disease appears to be spontaneous, although a history of trauma is not difficult to obtain in the first week of life, and it arises typically from the mucosa of the gastrointestinal tract. In addition to haematemesis and melaena there may be bruises in the skin (but no petechiae) and bleeding from the umbilicus and vagina. The onset of symptoms is between the second and seventh days but not after the seventh (except, perhaps, in premature babies) and the diagnosis is supported by a low prothrombin level and a prolonged coagulation time, the bleeding time and platelet count being normal. These tests do not prove the diagnosis, however, and as far as possible the many other causes of haemorrhage in the newborn must be excluded, mainly on clinical grounds. The following conditions must be considered:-

Birth trauma. 1

Asphyxia neonatorum.

Neonatal sepsis and septicaemia.

Neonatal vaginal bleeding.

Localized umbilical bleeding.

Haematemesis due to oesophagitis.
Congenital thrombocytopenic purpura. Afribrinogenaemia.

Of these, birth trauma and asphyxia are obvious, but since they both may cause intracranial haemorrhage, it may be impossible to make an exact diagnosis of the cause of this particular type of bleeding. Infections of various types are common in the newborn and, particularly when complicated by septicaemia, can cause widespread haemorrhage. This sort of bleeding is recognized by the local signs of the infection and by the blood culture, which is often positive. Neonatal vaginal bleeding, due in some way to the effect of maternal hormones, is only slight and is not accompanied by haemorrhage from other sites. When bleeding from the umbilicus is not part of a generalized haemorrhagic condition, it is due either to inefficient ligation of the cord or to a strictly localized infection of the cord stump. It is important to exclude haematemesis arising from local conditions in the oesophagus before attributing it to haemorrhagic disease. Even at this early age ulceration of the lower end of the oesophagus can occur as. a result of a congenital 'short oesophagus' with a partial intrathoracic stomach, and simple vomiting due to any cause may produce an

TABle I

Summary of the Diagnostic Features of the Haemorrhagic Diseases

\begin{tabular}{|c|c|c|c|c|c|c|c|c|}
\hline Disease & $\begin{array}{l}\text { Skin } \\
\text { Pur- } \\
\text { pura }\end{array}$ & $\begin{array}{l}\text { Tourni- } \\
\text { quet Test }\end{array}$ & $\begin{array}{l}\text { Platelet } \\
\text { Count }\end{array}$ & $\begin{array}{c}\text { Clot } \\
\text { Retraction }\end{array}$ & $\begin{array}{l}\text { Bleeding } \\
\text { Time }\end{array}$ & $\begin{array}{l}\text { Coagula- } \\
\text { tion Time }\end{array}$ & $\begin{array}{l}\text { Pro- } \\
\text { thrombin }\end{array}$ & $\begin{array}{l}\text { Other } \\
\text { Features }\end{array}$ \\
\hline $\begin{array}{l}\text { Hereditary } \\
\text { haemorrhagic } \\
\text { telangiectasia }\end{array}$ & No & Negative & Normal & Normal & Normal & Normal & Normal & $\begin{array}{c}\text { Hereditary } \\
\text { dominant } \\
\text { Both sexes }\end{array}$ \\
\hline Scurvy & Yes & Positive & Normal & Normal & Normal & Normal & Normal & \\
\hline $\begin{array}{l}\text { Anaphylactoid } \\
\text { purpura }\end{array}$ & Yes & $\begin{array}{c}\text { Sometimes } \\
\text { positive }\end{array}$ & Normal & Normal & Normal & Normal & Normal & \\
\hline $\begin{array}{l}\text { Idiopathic } \\
\text { thrombocyto- } \\
\text { penic purpura }\end{array}$ & Yes & Positive & Low & Abnormal & Prolonged & Normal & Normal & \\
\hline $\begin{array}{l}\text { Hereditary } \\
\text { haemorrhagic } \\
\text { thrombasthenia }\end{array}$ & $?$ & ? & $\begin{array}{c}\text { High } \\
\text { normal }\end{array}$ & $\begin{array}{c}\text { May be } \\
\text { abnormal }\end{array}$ & $\begin{array}{c}\text { May be } \\
\text { prolonged }\end{array}$ & Normal & Normal & $\begin{array}{l}\text { Hereditary } \\
\text { Both sexes }\end{array}$ \\
\hline Haemophilia & No & Negative & Normal & Normal & Normal & Prolonged & Normal & $\begin{array}{l}\text { Hereditary } \\
\text { recessive } \\
\text { Males only }\end{array}$ \\
\hline $\begin{array}{l}\text { Haiemorrhagic } \\
\text { disease of the } \\
\text { newborn }\end{array}$ & No & Negative & Normal & Normal & Normal & Prolonged & Low. & \\
\hline $\begin{array}{l}\text { Afibrino- } \\
\text { genaemia }\end{array}$ & No & Negative & Normal & 一 & Normal & - & Normal & $\begin{array}{l}\text { Absent } \\
\text { fibrinogen } \\
\text { No coagulation }\end{array}$ \\
\hline
\end{tabular}


oesophagitis with bleeding. Congenital thrombocytopenic purpura shows the same clinical features as the acquired type but occurs only when the disease has been present in the mother. Haemophilia, surprisingly, does not cause symptoms in the first week of life and afibrinogenaemia can easily be detected by examination of the blood (see Table I).

Of the other types of prothrombin deficiency little need be said. . Congenital idiopathic hypoprothrombinaemia has been described and, apart from an earlier onset of symptoms, produces a clinical picture identical with that of haemophilia. There is no family history but the coagulation time is prolonged, and if the prothrombin is not estimated these cases will be mistakenly diagnosed as sporadic haemophilia. Failure of absorption of vitamin $\mathrm{K}$ as in obstructive jaundice and chronic diarrhoea, or failure of formation of prothrombin in the liver in severe disease of that organ both result in hypoprothrombinaemia, but haemor $\cong$ rhagic symptoms are not a feature of these con- $-\frac{3}{\Phi}$ ditions.

Congenital afibrinogenaemia. About ten cases of $\stackrel{c}{\Rightarrow}$ congenital absence of fibrinogen have been re- $\overrightarrow{\vec{s}}$ corded. The disease is probably hereditary ando has the following features; a tendency to bleed듬 after minor injuries, from the time of birth; a complete absence of fibrinogen; failure of the $\mathbb{\Omega}$ blood to clot even after incubation; a very slow 0 sedimentation rate and a variable bleeding time. Diagnosis is therefore a simple matter once the $\vec{\circ}$ possibility of the disease has been considered.

I should like to thank Dr. W. G. Wyllie, Dr. Wilfrid Sheldon and Dr. Bernard Schlesinger foro permission to use photographs of their cases, which were kindly supplied by Mr. D. Martin.

I am greatly indebted to Dr. I. A. B. Cathie for $\stackrel{\omega}{\vec{H}}$ his helpful criticism.

\section{BIBLIOGRAPHY}

GAIRDNER, D. (1948), Quart. F. Med., 17, 95.

GLANZMANN, E. (1949), 'Einführung in die Kinderheilkunde,' 3rd Ed., Springer : Vienna.

LANCET (1950), ii, 690.

LEWIS, J. H., DAVIDSON, C. S., MINOT, G. R., SOULIER, J. P., TAGNON, H. J., and TAYLOR, F. H. L. (1946), $\mathscr{J}$. clin. Invest., 25, 870.

MACFARLANE, R. G. (1939), Lancet, i, 1199.

MACFARLANE, R. G. (1941), Quart. F. Med., 1o, I.
MERSKEY, C. (1950), f. clin. Path., 3, 130.

MERSKEY, C. (1951), Brit. Med. F., i, 906. QUICK, A. J. (I942), 'The Haemorrhagic Diseases,' Sprin。fie d, $\frac{}{\frac{D}{D}}$
Illinois: Charles C. 'Thomas.

QUICK, A. J. (1947), Amer. F. Med. Sci., 214, 272.

QUICK, A. J. (1949), Amer. F. Clin. Path., 19, ror6.

STEFANINI, M. (195I), Lancet, i, 606.

TOCANTINS, L. M. (1938), Medicine, Baltimore, $17 \times 55$. 